\title{
Crecimiento y reproducción de la mojarra amarilla (Caquetaia kraussii Steindachner, 1878) en el embalse de Urrá, Colombia
}

\author{
Growth and reproduction of yellow mojarra \\ (Caquetaia kraussii Steindachner, 1878) in the Urrá reservoir, \\ Colombia
}

Delio Solano-Peña, ${ }^{1,2}$ M.Sc, Fredys Segura-Guevara, ${ }^{2}$ M.Sc, Charles Olaya-Nieto, ${ }^{2 *}$ M.Sc.

${ }^{1}$ Autoridad Nacional de Acuicultura y Pesca. AUNAP. Bogotá, Colombia. ${ }^{2}$ Universidad de Córdoba, Facultad de Medicina Veterinaria y Zootecnia, Departamento de Ciencias Acuícolas, Laboratorio de Investigación Biológico Pesquera-LIBP. Lorica, Colombia. *Correspondencia: charles_olaya@hotmail. com

Recibido: Diciembre de 2011; Aceptado: Octubre de 2012.

\section{RESUMEN}

Objetivo. Evaluar los parámetros biológicos de crecimiento y reproducción de la mojarra amarilla en el embalse de Urrá. Materiales y métodos. Se colectaron 593 individuos para estudiar las relaciones talla-peso y la biología reproductiva. La relación longitud-peso y el factor de condición se estimaron con $\mathrm{WT}=\mathrm{a} \mathrm{LT}^{\mathrm{b}}$ y $\mathrm{Fc}=\mathrm{WT} / \mathrm{LT}^{\mathrm{b}}$, y se estimó proporción sexual, tallas e índices de madurez sexual, época de desove, diámetro de los ovocitos y fecundidad. Resultados. 235 individuos fueron hembras, 212 machos, 28 indiferenciados y 118 no sexados. La relación longitud-peso para sexos combinados fue $\mathrm{WT}=0.013( \pm 0.04) \mathrm{LT}^{3.07}( \pm 0.03), \mathrm{r}=0.99, \mathrm{n}=593$. La proporción sexual fue $1.1: 1$, la talla media de madurez sexual para sexos combinados fue $11.0 \mathrm{~cm}$ LT, el diámetro de los ovocitos fue $1376 \mu \mathrm{m}$ y la fecundidad promedio por desove fue 1732 ovocitos. Conclusiones. La mojarra amarilla mostró crecimiento isométrico en el embalse de Urrá, con talla media de captura menor que en el resto de la cuenca del río Sinú, sin dimorfismo sexual a la talla, período de reproducción prolongado y desoves parciales, ovocitos grandes y baja fecundidad, con correlación entre el factor de condición y el índice de madurez sexual, pero independientes del nivel de las aguas del embalse.

Palabras clave: Caquetaia kraussii, Colombia, crecimiento, reproducción (Fuente: AIMS). 


\section{ABSTRACT}

Objective. Assess the biological parameters of growth and reproduction of yellow mojarra of the Urrá reservoir. Materials and methods. 593 individuals were collected for this study where the lengthweight relationships and reproductive biology were evaluated. The length-weight relationship and condition factor were estimated through $T W=a T L^{b}$ and $C f=T W / T L^{b}$; sex ratio female: male, size and sexual maturity index, spawning season, oocyte diameter and fecundity were also estimated. Results. The study found 235 individuals were females, 212 were males, 28 were undifferentiated and 118

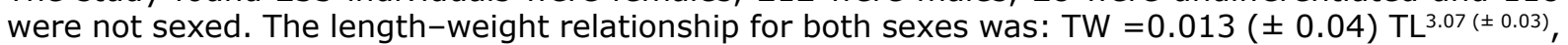
$r=0.99, n=593$. The sex ratio was $1.1: 1$, the length at first maturity for both sexes was $11.0 \mathrm{~cm} \mathrm{TL}$, oocyte diameter was $1376 \mu \mathrm{m}$ and average fecundity per spawn was 1732 oocytes. Conclusions. Yellow mojarra showed isometric growth in the Urrá reservoir, with an average catch size smaller than from the rest of the Sinu River basin, with no sexual dimorphism in size, prolonged spawning season and partial spawnings, large oocytes and low fertility, with a correlation between the condition factor and sexual maturity index, but independent of the water level of the reservoir.

Key words: Caquetaia kraussii, Colombia, growth, reproduction (Source: AIMS).

\section{INTRODUCCIÓN}

La Mojarra amarilla es nativa de Colombia y Venezuela, en la parte media y baja de los ríos Atrato, Sinú, San Jorge, Cesar, Arauca, Cauca, Magdalena, Catatumbo hasta Puerto Berrío. Presenta un color amarillo, con una serie de bandas transversales oscuras, manchas negras en la parte baja del opérculo, detrás de él, debajo de la aleta dorsal y una cuarta en la aleta caudal. Su hábitat son las aguas tranquilas de las tierras bajas (ciénagas), siempre y cuando sean aguas dulces o de salinidad muy baja (1).

Es una especie muy voraz, que consume peces, insectos y ovocitos, se reproduce durante todo el año presentando cuidado parental y que en la ciénaga Grande de Lorica no ha sido tan afectada por los cambios introducidos en la dinámica del río Sinú desde la construcción y puesta en marcha de la hidroeléctrica Urrá, convirtiéndose en especie reemplazo de las tradicionalmente capturadas y de mayor valor comercial de la cuenca, por lo que su pesquería no era tan crítica como la de otros peces, aunque debía regularse en el mediano plazo teniendo en cuenta que era la quinta especie en importancia comercial en la cuenca del río Sinú (2).

El objetivo de este proyecto fue evaluar los parámetros biológicos de crecimiento y reproducción de la Mojarra amarilla en el embalse de Urrá.

\section{MATERIALES Y MÉTODOS}

Localización y descripción del área de estudio. El área de estudio fue el embalse de Urrá, el cual se construyó represando al río Sinú a la altura de Angostura de Urrá a 267 km de su desembocadura, siendo desviado en el año 1995 e iniciándose su llenado a finales del año 1999 y su área de inundación es de 7400 ha. El embalse se ubica en el departamento de Córdoba, a $30 \mathrm{~km}$ del municipio de Tierralta. Los ríos Sinú y Verde confluyen en el embalse y son el principal aporte hidrológico al mismo, además de pequeñas quebradas que discurren aportando sus aguas al embalse (3). La pluviosidad en las zonas altas puede llegar hasta $2000 \mathrm{~mm}$ anuales en el embalse de Urrá y $5000 \mathrm{~mm}$ en las estribaciones del Nudo de Paramillo, mientras en las zonas bajas los valores medios anuales son de 1200 $\mathrm{mm}$ por año. Se observa un régimen bimodal de precipitaciones, con períodos lluviosos en abriljunio y agosto-octubre. El principal período seco se prolonga desde noviembre a marzo, con uno de menor proporción en julio-agosto (4).

Obtención de las muestras. La colecta de los individuos fue realizada por el Laboratorio de Investigación Biológico Pesquera-LIBP entre enero y diciembre 2003. Las muestras fueron tomadas de las capturas con los diferentes artes de pesca empleados en el embalse como son trasmallo y línea de mano. Se colectaron 593 individuos para la relación longitud-peso, a quienes se les tomó longitud total (LT) y longitud estándar (LS) al milímetro más cercano con un ictiómetro graduado en $\mathrm{mm}$ (Tridente, España), y el peso total (WT) al gramo más cercano con una balanza eléctrica (Ohaus, USA) con capacidad de $5000 \mathrm{~g}( \pm 1 \mathrm{~g})$.

Relaciones talla-peso. Se estimó la regresión lineal longitud estándar-longitud total ( $L T=a+b L S)$, aplicando el método de los mínimos cuadrados: $y=a+b x$ Ricker (5), en donde: $y$ es la variable dependiente medida en cm (LT), 
a es el intercepto de la recta de regresión, b es la pendiente de la recta de regresión y $\mathrm{x}$ es la variable independiente medida en $\mathrm{cm}$ (LS).

También se estimó la relación longitud-peso, la cual es una regresión potencial que relaciona una medida lineal (longitud) con una de volumen (peso) de acuerdo con la ecuación: $\mathrm{WT}=\mathrm{a} \mathrm{LT}^{\mathrm{b}}(6)$, en donde, WT es el peso total del pez en gramos, a es una constante de regresión equivalente al factor de condición (FC), LT es la longitud total del pez en centímetros y b es el coeficiente de crecimiento de la regresión. El factor de condición (Fc) se calculó mensual (enero a diciembre) y anual con la ecuación: $\mathrm{FC}=\mathrm{WT} / \mathrm{LT}^{\mathrm{b}}(6)$.

Los valores obtenidos para talla y peso total se expresaron como promedio ( \pm desviación estándar). Se establecieron intervalos de confianza al $95 \%$ y se aplicó el test de student al coeficiente de crecimiento de la relación longitudpeso para establecer la isometría. Se aplicó la prueba del análisis de varianza de una vía a la pendiente de la relación lineal, a los coeficientes de crecimiento de la relación longitud-peso y a los factores de condición mensuales. Cuando se encontraron diferencias estadísticas significativas se aplicó el test de comparaciones múltiples de Tukey-Kramer.

Biología reproductiva. Los peces se evisceraron y las gónadas se separaron del resto de órganos, se pesaron con una balanza eléctrica (Ohaus, USA) con capacidad de $1500 \mathrm{~g}( \pm 0.01) \mathrm{g}$, y se conservaron en solución de Gilson. Se registró la fecha, sitio de captura, arte de pesca utilizado, peso eviscerado, sexo, peso de las gónadas, número de la muestra y estado de madurez sexual de acuerdo con la siguiente escala: I, Inmaduro o Virgen; II, En maduración; III, Maduro y IV, Vacío o desovado (7).

La proporción sexual total se calculó con la ecuación: \%machos $=100 * \mathrm{Nm} / \mathrm{Nt}$ (8), en donde $\mathrm{Nm}$ es el número de machos y $\mathrm{Nt}$ el número total de individuos, y la proporción sexual a la talla con la técnica de Holden \& Raitt (9). Entre junio y diciembre se estimaron el Índice gonadosomático (IGS) (10): IGS $=100 *(W G / W T)$, en donde WG es el peso de las gónadas en gramos y WT es el peso total del pez en gramos; y el Índice gonadal (IG) (11): IG $=10^{n} *\left(W G / L T^{b}\right)$, en donde $L T$ es la longitud total del pez en centímetros, b es el coeficiente de crecimiento de la relación longitud-peso y $10^{\text {n }}$ es un factor utilizado para efectos de cálculos y comparación con IGS.

La talla media de madurez sexual se estimó con la metodología de Sparre \& Venema (12). Con un ocular micrométrico se midió el diámetro a 506 ovocitos procedentes de diferentes muestras de todos los meses en donde se colectó, seleccionadas al azar para observar y seleccionar el tamaño correspondiente a los ovocitos maduros lo cual está asociado con los estados de madurez sexual.

Para estimar la fecundidad por desove, se analizaron 29 muestras de hembras en estado III, tomándose tres submuestras con peso promedio de $0.15 \mathrm{~g}$ cada una por gónada. Se utilizó el método gravimétrico (9) con la ecuación: $F=n G / g$, en donde $n$ es el número de ovocitos maduros en la muestra, $G$ es el peso de todos los ovocitos y g es el peso de la muestra. Se utilizó estadística descriptiva y las variables fueron expresadas como promedio \pm desviación estándar. Para comprobar si la proporción sexual estimada se ajustaba a la esperada, se aplicó el test estadístico $\chi^{2}$-cuadrado.

\section{RESULTADOS}

Relaciones talla-peso. Se colectaron 593 individuos, de los cuales 235 fueron hembras, 212 machos, 28 indiferenciados y 118 individuos a los cuales no se les estableció el sexo. La longitud estándar osciló entre 5.8 y $27.0(10.2 \pm 4.0) \mathrm{cm}$ y coeficiente de variación de $39.5 \%$, la longitud total fluctuó entre 7.9 y $35.1(13.3 \pm 5.1) \mathrm{cm}$ y coeficiente de variación de $38.2 \%$ y el peso osciló entre 7.0 y $691.0(59.8 \pm 106.4) \mathrm{g}$ y coeficiente de variación de $181 \%$. Como todos los coeficientes de variación son mayores al $30 \%$, se infiere que las tallas y los pesos de la población son heterogéneos. La talla media de captura fue $13.3 \mathrm{~cm}$ LT, apreciándose que el $88.7 \%$ de los individuos $(n=526)$ son capturados con tallas menores a la talla mínima de captura permitida por el INPA (13) para la cuenca del Sinú $(17.1 \mathrm{~cm} \mathrm{LT}, 13.0 \mathrm{~cm} \mathrm{LS})$.

La regresión longitud estándar-longitud total anual estimada por sexo fue:

$$
\begin{aligned}
& \mathrm{LT}=0.46( \pm 0.14)+1.28( \pm 0.01) \mathrm{LS}, \mathrm{r}=0.99, \\
& \mathrm{n}=235(\text { Hembras }) \\
& \mathrm{LT}=0.47( \pm 0.13)+1.28( \pm 0.01) \mathrm{LS}, \mathrm{r}=0.99, \\
& \mathrm{n}=212(\text { Machos }) \\
& \mathrm{LT}=0.45( \pm 0.07)+1.28( \pm 0.01) \mathrm{LS}, \mathrm{r}=0.99, \\
& \mathrm{n}=593(\text { ambos sexos, Figura } 1)
\end{aligned}
$$

En la regresión longitud estándar-longitud total para sexos combinados se encontraron diferencias estadísticas significativas entre las pendientes estimadas para cada mes $(F=141.27$; $\mathrm{p}<0.0001 ; \mathrm{gl}=592)$. El test de Tukey-Kramer mostró que las diferencias se presentaron entre todos los meses evaluados. 


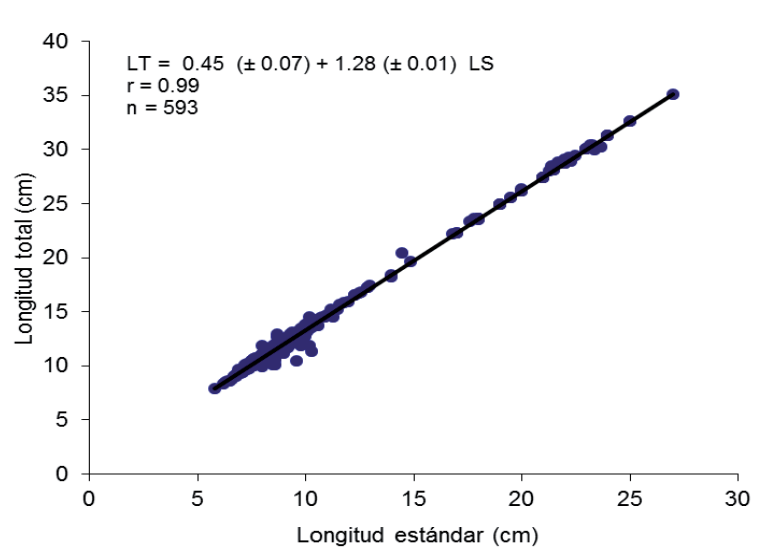

Figura 1. Relación longitud estándar-longitud total para sexos combinados de la Mojarra amarilla en el embalse de Urrá.

Los parámetros de crecimiento de la relación longitud-peso se estimaron a partir de la información mensual de longitud total y peso total, la cual se presenta en las tablas 1 y 2 . Para sexos combinados, el coeficiente de crecimiento b osciló entre 2.72 en diciembre y 3.33 en enero (Tabla 1), con valor anual de 3.07 (Tabla 2). El test de student $(p<0.05)$ confirmó que ocho coeficientes de crecimiento son isométricos o estadísticamente similares a tres $(b=3.0)$, mientras que el resto son estadísticamente diferentes de tres, tres alométricos positivos (b $>3.0$ ) (mayo, junio, septiembre) y uno alométrico negativo $(b<3.0)$ (diciembre), lo que indica que hubo correspondencia entre el crecimiento en longitud y el crecimiento en peso de la especie en ocho de los doce meses de estudio. Se encontraron diferencias estadísticas significativas entre los coeficientes de crecimiento mensuales ( $F=83.425 ; p<0.0001 ; g l=592)$. El test de Tukey-Kramer mostró que las diferencias se presentaron entre todos los meses evaluados. El valor anual de b para hembras (3.07), machos (3.08) y sexos combinados (3.07) fue isométrico $(=3.0)$.

El factor de condición osciló entre $0.006( \pm 0.71)$ en enero y $0.029( \pm 0.27)$ en diciembre (Tabla $2)$, siendo su valor anual $0.013( \pm 0.04)$, sin diferencias estadísticas significativas $(\mathrm{F}=0.1230$; $\mathrm{p}<0.0001 ; \mathrm{gl}=592$ ) y se confirmó la premisa de la relación inversa existente entre el coeficiente de crecimiento y el factor de condición.

A partir de las estimaciones del coeficiente de crecimiento y del factor de condición se construyeron las curvas anuales de la relación longitud-peso para hembras, machos y sexos combinados (Figura 2). Con referencia al ciclo hidrológico del embalse de Urrá, se observó correlación directa entre el factor de condición con los niveles del agua en mayo, junio, julio y agosto.

La regresión longitud total-peso total estimada fue:

WT $=0.013( \pm 0.09) L T^{3.07( \pm 0.09)}, r=0.98, n=$ 235 (hembras)

WT $=0.012( \pm 0.09) L T^{3.08( \pm 0.08)}, r=0.98, n=$ 212 (machos)

Tabla 1. Información básica de talla, peso y parámetros de crecimiento de la relación longitud-peso (LT-WT) en sexos combinados de la Mojarra amarilla. Año 2003.

\begin{tabular}{lccccccccccc}
\hline & & \multicolumn{2}{c}{ Longitud total $(\mathbf{c m})$} & \multicolumn{3}{c}{ Peso total (g) } & \multicolumn{3}{c}{ Relación longitud-peso } \\
\cline { 3 - 10 } \multicolumn{1}{c}{ Meses } & $\mathbf{n}$ & Rango & Prom. & D.E. & Rango & Prom. & D.E. & b \pm I.C. & a \pm I.C. & r & r ${ }^{2}$ \\
\hline Enero & 30 & $11.0-22.3$ & 13.3 & 2.4 & $9.0-173.0$ & 41.0 & 31.1 & $3.33 \pm 0.63$ & $0.006 \pm 0.71$ & 0.90 & 0.81 \\
Febrero & 28 & $10.5-13.5$ & 11.8 & 0.8 & $19.0-41.0$ & 27.1 & 6.1 & $2.80 \pm 0.57$ & $0.026 \pm 0.62$ & 0.89 & 0.79 \\
Marzo & 33 & $9.4-30.0$ & 14.6 & 6.1 & $14.0-453.0$ & 83.2 & 129.1 & $3.07 \pm 0.09$ & $0.013 \pm 0.11$ & 0.99 & 0.99 \\
Abril & 65 & $9.4-28.7$ & 13.3 & 4.3 & $13.0-375.0$ & 51.0 & 84.0 & $3.05 \pm 0.11$ & $0.013 \pm 0.12$ & 0.99 & 0.98 \\
Mayo & 56 & $8.6-35.1$ & 14.5 & 6.3 & $7.0-691.0$ & 83.7 & 149.2 & $3.16 \pm 0.08$ & $0.010 \pm 0.09$ & 0.99 & 0.99 \\
Junio & 77 & $7.9-33.3$ & 13.5 & 6.3 & $8.0-516.0$ & 73.4 & 130.5 & $3.06 \pm 0.05$ & $0.013 \pm 0.06$ & 0.99 & 0.99 \\
Julio & 61 & $9.0-31.3$ & 13.2 & 5.8 & $10.0-486.0$ & 62.4 & 115.7 & $3.04 \pm 0.07$ & $0.013 \pm 0.08$ & 0.99 & 0.99 \\
Agosto & 63 & $10.0-31.3$ & 13.2 & 5.0 & $15.0-486.0$ & 57.0 & 100.7 & $3.06 \pm 0.09$ & $0.013 \pm 0.09$ & 0.99 & 0.99 \\
Septiembre & 47 & $10.4-23.6$ & 12.1 & 2.6 & $15.0-214.0$ & 30.6 & 36.2 & $3.15 \pm 0.10$ & $0.010 \pm 0.11$ & 0.99 & 0.99 \\
Octubre & 40 & $8.5-30.4$ & 15.7 & 6.9 & $9.0-502.0$ & 96.8 & 136.5 & $3.06 \pm 0.09$ & $0.012 \pm 0.11$ & 0.99 & 0.99 \\
Noviembre & 47 & $8.7-32.6$ & 12.8 & 5.0 & $9.0-549.0$ & 53.9 & 121.7 & $3.03 \pm 0.15$ & $0.014 \pm 0.16$ & 0.99 & 0.97 \\
Diciembre & 46 & $9.0-17.2$ & 12.1 & 1.5 & $12.0-76.0$ & 26.7 & 12.0 & $2.72 \pm 0.25$ & $0.029 \pm 0.27$ & 0.96 & 0.92 \\
\hline
\end{tabular}

Tabla 2. Información básica anual de talla, peso y parámetros de crecimiento de la relación longitud-peso de la Mojarra amarilla. Año 2003.

\begin{tabular}{|c|c|c|c|c|c|c|c|c|c|c|c|}
\hline \multirow[b]{2}{*}{ Sexo } & \multirow[b]{2}{*}{$\mathbf{n}$} & \multicolumn{3}{|c|}{ Longitud total (cm) } & \multicolumn{3}{|c|}{ Peso total (g) } & \multicolumn{4}{|c|}{ Relación longitud-peso } \\
\hline & & Rango & Prom. & D.E. & Rango & Prom. & D.E. & b \pm I.C. & a \pm I.C. & $\mathbf{r}$ & $\mathbf{r}^{2}$ \\
\hline Hembras & 235 & $8.3-30.2$ & 12.2 & 3.1 & $9.0-502$ & 36.0 & 61.6 & $3.07 \pm 0.09$ & $0.013 \pm 0.09$ & 0.98 & 0.95 \\
\hline Machos & 212 & $7.9-30.4$ & 12.4 & 3.4 & $8.0-516$ & 39.0 & 74.0 & $3.08 \pm 0.08$ & $0.012 \pm 0.09$ & 0.98 & 0.96 \\
\hline SC & 593 & $7.9-35.1$ & 13.3 & 5.1 & $7.0-691$ & 58.7 & 106.4 & $3.07 \pm 0.03$ & $0.013 \pm 0.04$ & 0.99 & 0.98 \\
\hline
\end{tabular}




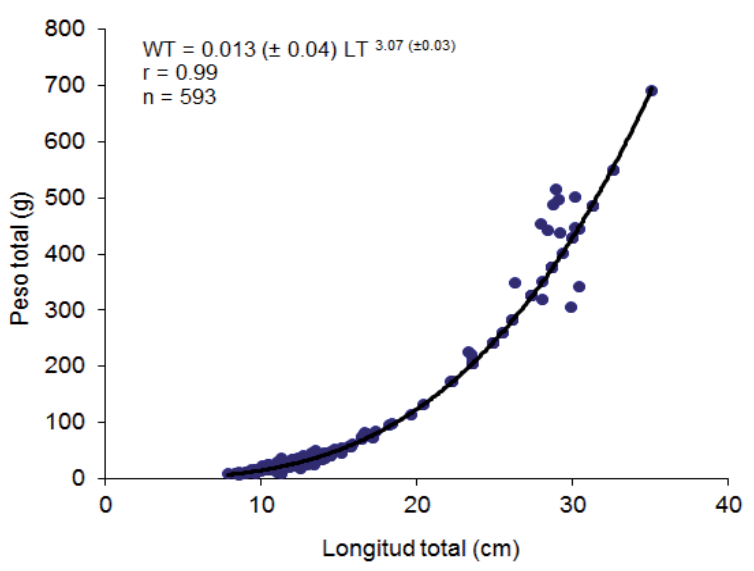

Figura 2. Relación longitud-peso de la Mojarra amarilla en el embalse de Urrá. Sexos combinados 2003.

WT $=0.013( \pm 0.04) \mathrm{LT}^{3.07( \pm 0.03)}, \mathrm{r}=0.99, \mathrm{n}=$ 593 (sexos combinados)

Biología reproductiva. De los 475 individuos que fueron sexados, 235 fueron hembras, 212 machos y 28 indiferenciados, notándose el alto número de indiferenciados en julio con $67.9 \%$ del total. Las tallas oscilaron entre 7.9 y 30.4 (12.2 \pm 3.2$) \mathrm{cm}$ LT con coeficiente de variación de $25.9 \%$ (homogéneo) y los pesos totales

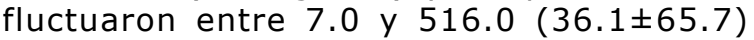
g con coeficiente de variación de $181.9 \%$ (heterogéneo).

La proporción sexual hembra: macho observada fue $1.1: 1$, similar a lo esperado $\left(\chi^{2}: 1.183\right.$; $\mathrm{p}<0.05 ; 1 \mathrm{gl})$. La proporción sexual hembra: macho a la talla fue similar a lo esperado en casi todos los intervalos excepto entre $17.0-19.0 \mathrm{~cm}$ LT $\left(\chi^{2}: 4.000 ; p<0.05 ; 1 \mathrm{gl}\right)$. En ambos casos, ya sea para meses como para tallas, se observa que en el $91.7 \%$ hay correspondencia con la proporción sexual total.

El $41.5 \%$ de los individuos colectados $(n=197)$ fueron individuos inmaduros o vírgenes o en estado de madurez I, seguidos por individuos maduros en estado de madurez III con $30.1 \%$ $(n=143)$. La colecta de ejemplares hembras maduras se dió en casi todos los meses del año $(n=57)$, excepto abril, resaltando que fue casi el $50 \%$ de los individuos inmaduros o vírgenes. Mayo y junio fueron los meses con mayor número de individuos colectados. Para machos se observaron individuos maduros en diez meses del año ( $n=86)$, excepto enero y abril, siendo equivalente al número de individuos inmaduros o vírgenes colectados $(n=86)$. Mayo (17), junio (17) y julio (11) fueron los meses con mayor número de individuos colectados, respectivamente (Tabla 3 ). Para las hembras
Tabla 3. Número mensual de estadios de madurez gonadal de la Mojarra amarilla en el embalse de Urrá. Año 2003.

\begin{tabular}{lcccccccccc}
\hline \multicolumn{1}{c}{ Mes } & \multicolumn{4}{c}{ Hembras } & \multicolumn{5}{c}{ Machos } \\
\hline Enero & I & II & III & IV & Total & I & II & III & IV & Total \\
Febrero & 8 & 4 & 2 & 0 & 14 & 2 & 6 & 0 & 0 & 8 \\
Marzo & 6 & 2 & 6 & 1 & 15 & 7 & 2 & 4 & 0 & 13 \\
Abril & 12 & 2 & 3 & 0 & 17 & 7 & 1 & 4 & 0 & 12 \\
Mayo & 28 & 8 & 0 & 0 & 36 & 17 & 4 & 0 & 0 & 21 \\
Junio & 4 & 6 & 13 & 2 & 25 & 4 & 1 & 17 & 0 & 22 \\
Julio & 10 & 12 & 8 & 2 & 32 & 11 & 1 & 17 & 2 & 31 \\
Agosto & 3 & 7 & 5 & 1 & 16 & 5 & 3 & 11 & 0 & 19 \\
Septiembre & 4 & 4 & 2 & 1 & 13 & 2 & 2 & 4 & 0 & 8 \\
Octubre & 3 & 3 & 4 & 0 & 10 & 3 & 2 & 9 & 0 & 14 \\
Noviembre & 9 & 4 & 3 & 0 & 16 & 14 & 8 & 7 & 0 & 29 \\
Diciembre & 18 & 2 & 5 & 0 & 25 & 8 & 4 & 9 & 0 & 21 \\
Total & $\mathbf{1 1 1}$ & $\mathbf{6 0}$ & $\mathbf{5 7}$ & $\mathbf{7}$ & $\mathbf{2 3 5}$ & $\mathbf{8 6}$ & $\mathbf{3 8}$ & $\mathbf{8 6}$ & $\mathbf{2}$ & $\mathbf{2 1 2}$ \\
\hline
\end{tabular}

maduras en estado de madurez gonadal II y III, el índice gonadosomático osciló entre 0.447-5.833 (2.758 \$1.33). A nivel mensual, este índice fluctuó entre 0.946 (noviembre) y 3.706 (julio), observándose valores alternantes mientras que el nivel del embalse aumentaba paulatinamente entre junio y diciembre (Figura 3). Lo anterior indica que el desove de la especie no está sujeto o influenciado por los niveles del

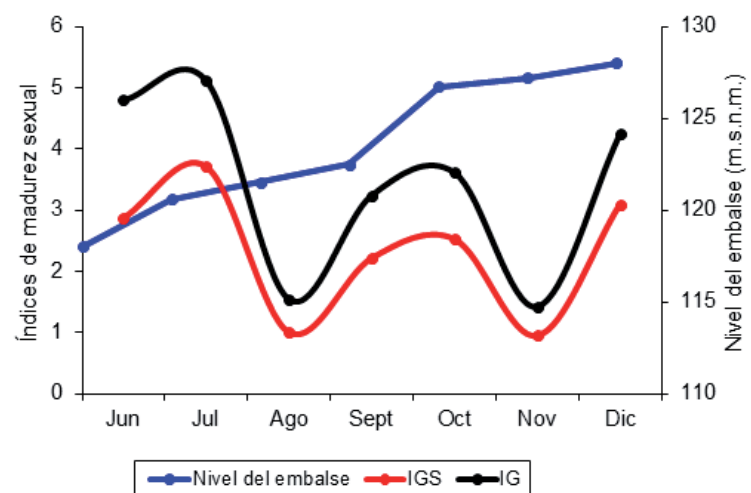

Figura 3. Índices de madurez sexual de hembras de Mojarra amarilla en el embalse de Urrá. Año 2003.

cuerpo de agua en que habita, a diferencia de los peces reofílicos.

La talla de inicio de madurez sexual encontrada fue de $9.4 \mathrm{~cm} \mathrm{LT}(7.0 \mathrm{~cm} \mathrm{LS})$ y $9.6 \mathrm{~cm} \mathrm{LT}(7.1 \mathrm{~cm}$ LS) para hembras y machos, respectivamente. La talla media de madurez sexual fue estimada en $10.4,11.5$ y $11.0 \mathrm{~cm}$ LT $(7.8,8.6$ y $8.2 \mathrm{~cm}$ LS) para hembras, machos y sexos combinados (Figura 4), respectivamente. El diámetro de los maduros fluctuó entre 1282 y $1619 \mu \mathrm{m}$, con promedio de $1376 \pm 75 \mu \mathrm{m}$, correspondiendo a ovocitos grandes. 


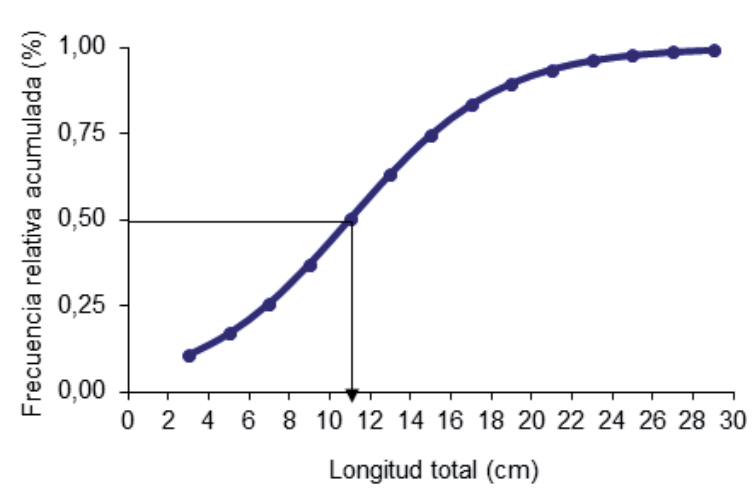

Figura 4. Talla media de madurez sexual para sexos combinados de Mojarra amarilla en el embalse de Urrá. Año 2003.

La colecta de hembras y machos en estado de madurez III en casi todos los meses del año, los índices de madurez gonadal y el diámetro de los ovocitos maduros permitió inferir que la Mojarra amarilla es un pez con ovocitos grandes y un período de reproducción prolongado durante el año, en el que se presentan varios desoves parciales con evento principal en julio cuando los índices de madurez gonadal alcanzaron sus máximos valores. La fecundidad promedio por desove o número de ovocitos promedio por desove fue estimada en $1732 \pm 2368$.

\section{DISCUSIÓN}

A pesar de las desviaciones observadas en las longitudes estándar y total de los peces muestreados y de que los coeficientes de variación fueros heterogéneos, no se observó dimorfismo sexual a la talla al no encontrarse estadísticamente predominio de un sexo sobre el otro, a diferencia de lo reportado para la especie en las ciénagas Grande de Lorica y de Ayapel (14). Cabe anotar que las diferencias en la tasa de crecimiento entre hembras y machos pueden estar relacionadas con las proporciones corporales $\mathrm{y}$, como tal, pueden traducirse en dimorfismo sexual debido a sus estrategias reproductiva, trófica y de comportamiento, y al patrón de gasto de energía en el mantenimiento corporal (15).

Según diferentes estudios, la talla media de captura para la Mojarra amarilla en la cuenca del río Sinú ha fluctuado entre $14.0 \mathrm{~cm} \mathrm{LS}, 18.3 \mathrm{~cm}$ LT y $15.3 \mathrm{~cm}$ LS, $19.9 \mathrm{~cm} \mathrm{LT}$, mientras que en el embalse de Urrá, ha oscilado entre $10.8 \mathrm{~cm}$ LS, $14.6 \mathrm{~cm}$ LT y $14.8 \mathrm{~cm}$ LS, $19.3 \mathrm{~cm}$ LT (16). Todas las tallas medias de captura encontradas por los autores citados arriba para la especie en la cuenca del río Sinú, son mayores que la estimada en este trabajo ( $13.3 \mathrm{~cm} \mathrm{LT})$, lo cual puede estar relacionado con la ubicación geográfica de las áreas estudiadas, la selectividad del arte de pesca utilizado en los diferentes estudios, la presión pesquera ejercida sobre el recurso, el número de individuos colectados y las condiciones ambientales que presenta el embalse. Además, la mayoría de los peces en los ambientes tropicales muestra gran flexibilidad alimentaria (17), plasticidad trófica que puede ser una estrategia importante para el uso del abundante recurso alimenticio disponible al inicio de la formación, construcción o llenado de un reservorio o embalse.

No obstante las diferencias descritas entre las tallas y pesos colectados en este estudio con las tallas y pesos diferentes trabajos, es importante comentar la similitud encontrada entre los coeficientes de crecimiento estimados con el reportado para la especie (3.01) en la ciénaga Grande de Lorica (2), siendo todos isométricos. Dado que la talla es una magnitud lineal y el peso es proporcional a su cubo, a lo largo de su crecimiento la mayor parte de los peces tienen dimensiones diferentes. Los coeficientes de crecimiento estimados en este trabajo para hembras (3.07), machos (3.08) y sexos combinados (3.07) se encuentran dentro del rango 2.73-3.11, cuyo promedio \pm intervalo de confianza al $99 \%$ es de $2.92( \pm 0.19)$, de acuerdo con diferentes reportes $(18-22,2,14)$ para algunas especies de la familia Cichlidae (Tabla 4).

La correlación encontrada entre el factor de condición con los niveles del agua a lo largo del año de estudio, especialmente en aguas altas, puede atribuirse a que en esa época hay una mayor disponibilidad de alimento, debido principalmente a la incorporación de material biológico terrestre al medio acuático (23) y al incremento de la producción en todos los niveles tróficos (24), por lo que la Mojarra amarilla, como muchos otros peces, puede responder a dicha disponibilidad de alimento con un mayor consumo que se traduce en el incremento del factor de condición. Sin embargo, en ningún caso dicha correlación fue estadísticamente significativa al $95 \%$ de confianza, por lo que puede afirmarse que a lo largo del ciclo hidrológico no se encontró un patrón de correlación claro y definido entre estas dos variables, posiblemente porque la Mojarra amarilla es un pez sedentario cuyo ciclo de vida (crecimiento y reproducción, por ejemplo) no está sujeto o es poco influenciado por el ciclo hidrológico del cuerpo de agua en que habite.

La proporción sexual típica en peces es de $1: 1$ y las explicaciones se dan en términos del gasto en que incurre cada sexo en el proceso reproductivo (25), en donde encaja la Mojarra 
Tabla 4. Parámetros de crecimiento de la relación longitud-peso de la Mojarra amarilla y algunas especies de la familia Cichlidae en América del Sur.

\begin{tabular}{lcccccc}
\hline \multicolumn{1}{c}{ Especie } & Medición (cm) & $\mathbf{a}$ & $\mathbf{b}$ & $\mathbf{n}$ & $\mathbf{r}$ & Fuente \\
\hline Crenicichla britskii - SC & LS & 0.1039 & 2.34 & 64 & 0.94 & Benedito-Cecilio et al (18) \\
Crenicichla haroldoi - SC & LS & 0.037 & 2.71 & 43 & 0.96 & Benedito-Cecilio et al (18) \\
Crenicichla nierderleinii - SC & LS & 0.033 & 2.74 & 343 & 0.96 & Benedito-Cecilio et al (18) \\
Geophagus brasiliensis - SC & LS & $4.2 \times 10-5$ & 2.85 & 149 & 0.99 & Santos et al (19) \\
Geophagus brasiliensis - SC & LT & 0.025 & 2.93 & 883 & - & Moraes et al (22) \\
Satanoperca pappaterra - SC & LS & 0.0284 & 3.13 & 153 & 0.99 & Benedito-Cecilio et al (18) \\
Andinoacara pulcher - SC & LT & 0.023 & 3.01 & 298 & 0.94 & Olaya-Nieto et al 2005 (datos no pub.) \\
Andinoacara pulcher - SC & LT & 0.024 & 2.99 & 455 & 0.94 & Olaya-Nieto et al (14) \\
Andinoacara pulcher - H & LT & 0.022 & 3.04 & 259 & 0.94 & Olaya-Nieto et al (14) \\
Andinoacara pulcher - M & LT & 0.028 & 2.94 & 192 & 0.93 & Olaya-Nieto et al (14) \\
Caquetaia kraussii - SC & LS & $1 \times 10-5$ & 3.18 & 563 & 0.99 & Valderrama et al (21) \\
Caquetaia kraussii - SC & LS & $2 \times 10-5$ & 3.13 & 583 & 0.97 & Valderrama et al (21) \\
Caquetaia kraussii - SC & LT & 0.017 & 3.01 & 3082 & 0.96 & Olaya-Nieto et al (2) \\
Caquetaia kraussii - SC & LT & 0.013 & 3.07 & 593 & 0.99 & Este trabajo \\
Caquetaia kraussii - H & LT & 0.012 & 3.08 & 235 & 0.97 & Este trabajo \\
Caquetaia kraussii - M & LT & 0.013 & 3.07 & 212 & 0.98 & Este trabajo
\end{tabular}

$\mathrm{SC}=$ sexos combinados, $\mathrm{H}=$ hembra, $\mathrm{M}=$ macho, $*$ talla en $\mathrm{mm}$.

amarilla. Además, la proporción sexual en los peces varía considerablemente de una especie a otra, y puede diferir de una población a otra, e incluso- variar de un año a otro dentro de la misma población (26). La mortalidad y el crecimiento diferencial entre ambos sexos afectan la proporción sexual (11), debido a la selectividad que introduce el arte de pesca que se utilice en la pesquería de la especie y de la población (14).

La talla media de madurez sexual estimada para hembras, machos y sexos combinados es menor que la talla mínima de captura $(17.1 \mathrm{~cm} \mathrm{LT}, 13.0$ $\mathrm{cm}$ LS, ) recomendada por el INPA (13) para la cuenca del río Sinú, con base en estimaciones realizadas en la ciénaga Grande de Lorica, cuerpo de agua con condiciones muy diferentes al embalse de Urrá. Olaya-Nieto et al. (2) estimaron en 17.8, 17.9 y $17.8 \mathrm{~cm}$ LT las tallas medias de madurez sexual para hembras, machos y sexos combinados de individuos de Mojarra amarilla, tallas que son mucho mayores porque provienen de la pesquería comercial de la ciénaga Grande de Lorica, cuenca Baja del río Sinú, y que presentan -ademásdiferencias estadísticas significativas con las tallas colectadas en este trabajo.

En el Acuerdo 007 del Incoder se establecen tallas mínimas de captura para cinco especies Prochilodus magdalenae, Sorubim cuspicaudus, Ageneiosus pardalis, Brycon sinuensis y Salminus affinis, en el embalse de Urrá, excluyendo a la especie en estudio, por lo que se recomienda a las autoridades pesqueras y ambientales adoptar una talla mínima de captura para la Mojarra amarilla, ya sea de 12.2 $\mathrm{cm}$ LT $09.2 \mathrm{~cm}$ LS.

En general, los ovocitos son grandes y ovalados, similares a los de Cocobolo (Andinoacara pulcher) y a los de otras especies y géneros de la familia Cichlidae, concordando con lo reportado por otros autores y se ubican dentro de los cinco de mayor tamaño encontrados en los peces nativos de la cuenca del río Sinú, siendo su diámetro similar al de los ovocitos maduros $(1388 \mu \mathrm{m})$ de individuos colectados en las faenas de la pesquería comercial de la ciénaga Grande de Lorica (2).

En cuanto a la época de desove, un largo período reproductivo es una característica de los peces neotropicales y constituye un componente crítico en el ciclo de vida de un organismo (26), que puede ser influenciado por el ambiente físico, la disponibilidad de alimento y los factores bióticos (27). Para la Mojarra amarilla, los factores que desencadenan su reproducción no son muy evidentes, debido a que la especie se reproduce durante todo el año, a pesar de mostrar un pico de desove en la época de lluvias (julio). El desove parcial o múltiple puede ser entendido como un mecanismo para compensar la alta mortalidad al inicio del ciclo de vida de las especies de peces con baja fecundidad (28) como la Mojarra amarilla.

Este tipo de desove es de fundamental importancia para los peces pequeños porque les permite un aumento considerable en la fecundidad, lo cual no sería posible de otro modo debido a las limitaciones de tamaño, lo que resulta en una mayor probabilidad de sobrevivencia de la prole por el desove de un mayor número de ovocitos, lo que explicaría la abundancia de la especie (26). Los resultados obtenidos en este trabajo concuerdan con lo afirmado por otros autores $(2,29)$, quienes encontraron que la especie en estudio se reproduce durante todo el año en el lago de Valencia (Venezuela) y en la ciénaga Grande de Lorica, respectivamente. 
La fecundidad promedio por desove es diferente a la fecundidad absoluta de 5570 ovocitos (29) y mayor que la fecundidad por desove reportada para algunas especies muy cercanas de la familia Cichlidae como Aequidens latifrons (800 ovocitos) (30), A. tetramerus (1000 ovocitos) (31) y Cocobolo Andinoacara pulcher (954 ovocitos) (32). Dicha fecundidad puede variar entre períodos de reproducción consecutivos y entre individuos de la misma talla en el mismo período reproductivo, que -además- de las variaciones interespecíficas, puede ser una característica inestable (11) y también puede variar según la disponibilidad de alimento, el tipo de desove, la edad de primera madurez, la temperatura, la latitud y el cuidado parental (26).

A pesar de estas variaciones, parece haber un consenso en torno a la baja fecundidad en especies de pequeño tamaño, con desove parcial o múltiple, no migradoras o sedentarias, con época de desove prolongada, cuidado parental y ovocitos grandes (33), como la Mojarra amarilla, especie que presenta una estrategia reproductiva del tipo $\mathrm{r} 1$, la cual se caracteriza por baja sobrevivencia en las fases iniciales del ciclo de vida (larva a juvenil) y en la fase adulta, baja fecundidad pero con repetidos eventos de desove, ciclo de vida corto y densidad poblacional variable a través del año (34).

Por todo lo anterior, se concluye que la Mojarra amarilla presenta un crecimiento isométrico en el embalse de Urrá, con talla media de captura menor que en el resto de la cuenca del río Sinú, sin presentar dimorfismo sexual a la talla, cuya época o período de reproducción se prolonga durante el año con desoves parciales, ovocitos grandes y baja fecundidad, con correlación entre el factor de condición y el índice de madurez sexual, pero independientes del nivel de las aguas del embalse.

\section{Agradecimientos}

A la Oficina de Investigación y Extensión de la Universidad de Córdoba, por la financiación del proyecto de investigación "Estimación de los parámetros biológicos básicos de peces comerciales del Río Sinú - Fase III", Código FMV-01-06, Numeral 1.2.08.031, del cual hace parte este trabajo; y a los pescadores de la cuenca del río Sinú.

\section{REFERENCIAS}

1. Dahl G. Los peces del norte de Colombia. Bogotá: Inderena; 1971.

2. Olaya-Nieto CW, Brú-Cordero SB, SeguraGuevara F, Tordecilla-Petro G. Estimación de los parámetros biológicos básicos de peces comerciales del Río Sinú-Fase I. Informe final. Lorica: Laboratorio de Investigación Biológico Pesquera-LIBP, Departamento de Acuicultura, Facultad de Medicina Veterinaria y Zootecnia, Universidad de Córdoba; 2004.

3. Urrá SA ESP. Caracterización socioeconómica de las comunidades de pescadores. Tierralta: Proyecto Monitoreo pesquero del embalse; 2001.

4. IDEAM. Base de datos. Barranquilla; 1998.

5. Ricker WE. Computation and interpretation of biological statistics of fish population. J Fish Res Board Can 1975; 191:1-382.

6. Bagenal TB, Tesch FW. Age and growth. In: Bagenal TB (ed.). Methods for assessment of fish production in fresh waters. IBP Handbook No. 3. Oxford: Blackwell Scientific Publications; 1978.

7. Vazzoler AEAM. Diversificação fisiológica e morfológica de Micropogonias furnieri (Desmarest, 1822) ao sul do Cabo Frio, Brasil. Bol Inst Oceanogr 1971; 20(2):1-20.
8. Wenner AM. Sex ratio as a function of size in marine crustacea. Amer Nat 1972; 106(949): 321-350.

9. Holden MJ, Raitt DFS. Manual de ciencias pesqueras. Parte 2. Métodos para investigar los recursos y su aplicación. FAO Doc Téc Pesca $1975 ; 115: 1-211$.

10. Vazzoler AEA de M, Caraciolo-Malta MC, Amadio SA. Aspectos biológicos de Peixes amazônicos. XII. Indicadores quantitativos do período de desova das espécies da gênero Semaprochilodus (Characiformes, Prochilodontidae) do baixo rio Negro, Amazonas, Brasil. Rev Bras Biol 1989; 49(1): 175-181.

11. Vazzoler AEAM. Biologia da reprodução de peixes teleósteos: teoria e prática. Maringá: EDUEM; 1996.

12. Sparre $P$, Venema SC. Introducción a la evaluación de recursos pesqueros tropicales. Parte 1. Manual. FAO Doc Téc Pesca 1995; 306/1(rev.1):1-420.

13. INPA. Resolución 00520 de noviembre 8 del 2001. Bogotá. 2001; 3.

14. Olaya-Nieto CW, Segura-Guevara FF, TordecillaPetro G, Appeldoorn RS. Estimación de los 
parámetros biológicos básicos de peces comerciales del Río Sinú-Fase III. Informe final. Lorica: Laboratorio de Investigación Biológico Pesquera-LIBP, Programa de Acuicultura, Departamento de Ciencias Acuícolas, Facultad de Medicina Veterinaria y Zootecnia, Universidad de Córdoba; 2011.

15. Orsi ML, Shibatta OA. Crescimento de Schizodon intermedius Garavello and Britski (Osteichthyes, Anostomidae) do rio Tibagi (Sertanópolis, Paraná). Rev Bras de Zool 1999; 13(3):701-7.

16. Valderrama M, Salas F, Solano D. Los peces y las pesquerías en el embalse de Urrá 2001-2005. Montería: Fundación Bosques y Humedales-Urrá S.A. E.S.P.; 2006.

17. Lowe-McConnell RH. (ed.). Estudos ecológicos de comunidades de peixes tropicais. Tradução: Vazzoler AEA de M, Agostinho AA, Cunningham PTM. (eds.). São Paulo: EDUSP; Ecological studies in tropical fish communities: 1999.

18. Benedito-Cecilio E, Agostinho AA, Velho RCCM. Length-weight relationship of fishes caught in the Itaipu Reservoir, Paraná, Brazil. Naga ICLARM Quart 1997; 20(3-4):57-61.

19. Santos AFGN dos, Santos LN dos, Araújo FG, Santos RN dos, Andrade CC de, Silva PS, et al. Relação peso-comprimento e fator de condição do acará; Geophagus brasiliensis, no reservatório de Lajes, RJ. Rev Univ Rural Sér Ciên da Vida 2002; 22(2):115-121.

20. Valderrama M, Garzón AC, Salas F, Villadiego $P$, Rangel $B$. Monitoreo ictiológico y pesquero del embalse de Urrá. Montería: Informe final año 2001 presentado a Urrá S.A. E.S.P.; 2002.

21. Valderrama M, Garzón AC, Salas F, Rangel $B$, Solano D, Fadul M. Monitoreo ictiológico y pesquero del embalse de Urrá. Montería: Informe final año 2002 presentado a Urrá S.A. E.S.P.; 2003.

22. Moraes MFPG, Barbola IF, Duboc LF. Feeding habits and morphometry of digestive tracts of Geophagus brasiliensis (Osteichthyes, Cichlidae), in a lagoon of high Tibagi River, Paraná State, Brazil. Publ UEPG Ci Biol Saúde 2004; 10(1):37-45.

23. Agostinho AA, Miranda LE, Bini LM, Gomes LC, Thomaz SM, Suzuki HI. Patterns of colonization in neotropical reservoirs, and prognoses on aging. In: Tundisi JG, Straskrăba M. (eds.). Theoretical reservoir ecology and its applications. Leiden: Backhuys Publishers; 1999: 227-265.

24. O'Brien WJ. Perspectives on fish in reservoir limnology. In: Thornton KW, Kimmel BL, Payne FE. (eds.). Reservoir limnology: ecological perspectives. New York: John Wiley and Sons; 1990.

25. Krebs JR, Davies NB. Introdução à ecologia comportamental. São Paulo: Atheneu Editora; 1996.

26. Nikolsky GV. The ecology of fishes. London: Academic Press; 1963.

27. Kramer DL. Reproductive seasonality in the fishes of a tropical stream. Ecology 1978; 59(5):976-985.

28. Vazzoler AEAM, Menezes NA. Síntese de conhecimentos sobre o comportamento reprodutivo dos Characiformes da América do Sul (Teleostei, Ostariophysi). Rev Bras Biol 1992; 52(4):627-640.

29. Lasso CA, Machado-Allison A. Sinopsis de las especies de peces de la familia Cichlidae presentes en la cuenca del río Orinoco. Caracas: Museo de Historia Natural La Salle, Instituto de Zoología Tropical, Universidad Central de Venezuela; 2000.

30. Stawikowski $R$, Werner O. Die Buntbarsche Amerikas, Band 1. Stuttgart: Verlag Eugen Ulmer; 1998.

31. Planquette $P$, Keith $P$, Le Bail PY. Atlas des poissons d'eau douce de Guyane (Tome 2). Paris: Publications Scientifiques du Muséum National d'Histoire Naturelle, Collection du Patrimoine Naturel; 2000.

32. Olaya-Nieto CW, Bautista-Blanco AL, PérezPisciotti M. Biología reproductiva del Cocobolo (Andinoacara pulcher Musilová et al., 2009) (Pisces: Cichlidae) en la ciénaga Grande de Lorica (Córdoba), Colombia. Actual Biol 2010; 32(92):65-73.

33. Araújo RB, Garutti V. Biologia reprodutiva de Aspidoras fuscoguttatus (Siluriformes, Callichthyidae) em um riacho de cabeceira da bacia do Alto Rio Paraná. Iheringia Série Zoologia 2002; 92(4):89-98.

34. Winemiller KO, Taphorn DC. La evolución de las estrategias de vida en los peces de los llanos occidentales de Venezuela. Biollania $1989 ; 6: 77-122$. 